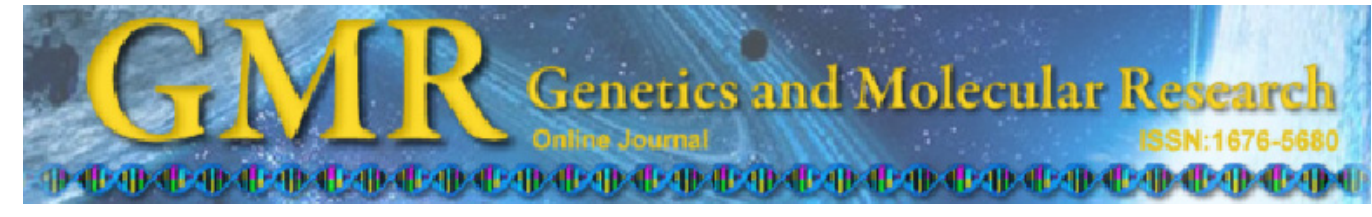

\title{
Methylenetetrahydrofolate reductase genotypes and haplotypes associated with susceptibility to colorectal cancer in an eastern Chinese Han population
}

\author{
H. Li, W.L. Xu, H.L. Shen, Q.Y. Chen, L.L. Hui, L.L. Long and X.L. Zhu \\ Department of Central Laboratory, The Affiliated People's Hospital, \\ Jiangsu University, Zhenjiang, Jiangsu, China \\ Corresponding author: W.L. Xu \\ E-mail: xuw10511@yahoo.com
}

Genet. Mol. Res. 10 (4): 3738-3746 (2011)

Received May 18, 2011

Accepted November 9, 2011

Published December 14, 2011

DOI http://dx.doi.org/10.4238/2011.December.14.8

\begin{abstract}
Methylenetetrahydrofolate reductase (MTHFR) plays an important role in folate metabolism and is involved in DNA synthesis, DNA repair and DNA methylation. The two common functional polymorphisms of MTHFR, C677T and A1298C have been associated with several diseases, including cancer. We made a case-control study to analyze a possible association of MTHFR gene polymorphisms C677T and A1298C with risk for colorectal cancer in an eastern Chinese Han population of 137 patients with a confirmed histopathological diagnosis of CRC and 145 age- and gender-matched controls with no history of cancer. DNA was isolated from peripheral blood samples and the genotypes were determined by PCR-RFLP. The concentrations of folate in plasma were measured by chemiluminescence immunoassay. The MTHFR 677TT genotype had a protective effect against colorectal cancer, with an odds ratio $(\mathrm{OR})=0.467(95 \%$ confidence interval $(\mathrm{CI})=$ $0.225-0.966)$. The $1298 \mathrm{CC}$ genotype was significantly correlated with a reduced risk of colorectal cancer $(\mathrm{OR}=0.192 ; 95 \% \mathrm{CI}=0.040-0.916)$. Compared with the MTHFR 677CC and MTHFR 1298 AA genotypes,
\end{abstract}


for individuals who carried both MTHFR 677CC and 1298CC genotypes, the OR of colorectal cancer was $0.103(95 \% \mathrm{CI}=0.012-0.900)$; among individuals who carried both MTHFR 677TT and 1298AC genotypes, the OR for risk of colorectal cancer was $0.169(95 \% \mathrm{CI}=0.044-0.654)$. MTHFR 677TT+CT genotypes had a significantly lower plasma folate concentration than those with the MTHFR 677CC genotype. MTHFR $1298 \mathrm{AC}+\mathrm{CC}$ genotypes had a lower plasma folate concentration than those with the MTHFR 1298AA genotype $(\mathrm{P}<0.05)$. In conclusion, subjects with the MTHFR 677TT and MTHFR 1298CC genotypes appeared to have a significantly lower risk for colorectal cancer. MTHFR haplotypes 677CC/1298CC and 677TT/1298AC were less common in cases than in controls. These haplotypes, when compared to the most common haplotype $677 \mathrm{CC} / 1298 \mathrm{AA}$, were associated with a decreased risk for colorectal cancer. We conclude that plasma folate level is influenced by MTHFR genotypes.

Key words: Colorectal cancer risk; MTHFR; Genotype; Haplotype

\section{INTRODUCTION}

Colorectal cancer (CRC) is a common malignancy and one of the leading causes of death in Western societies (Jemal et al., 2007; Coleman et al., 2008). It is the second most common cause of death associated with all cancer types in men and women in Europe (Boyle and Ferlay, 2005). Studies have found that colorectal cancer is also the most common cancer in developed countries (Parkin et al., 2001). Worldwide, colorectal cancer represents $9.4 \%$ of all incidents of cancer in men and $10.1 \%$ in women (Boyle and Langman, 2000). It is estimated that over half the people diagnosed with colorectal cancer will die of the disease (Boyle and Ferlay, 2005). Colorectal cancer is very common and has increased rapidly along with the westernization of lifestyle in China. The data from the municipal death registry system in cities of China show that the death rate of CRC has increased in recent decades and that CRC is becoming a growing health problem in cities of China (Song et al., 2008). The epidemiological studies of sporadic CRC have established that many etiologic factors, including age, sex, smoking status, alcohol consumption and family history of cancer, may influence the risk of developing cancer and its clinical outcome (Wei et al., 2009). Aside from the traditional risk factors, an effort has been made to detect genetic factors that contribute to the risk of colorectal cancer development. Epidemiological studies have indicated an association between folate intake and the risk of certain cancers (Eichholzer et al., 2001; La Vecchia et al., 2002; Larsson et al., 2006a,b), including colorectal cancer. Folate deficiencies may result in abnormal DNA methylation and uncontrolled gene expression leading to malignant transformation (Duthie, 1999; Choi and Mason, 2000). Polymorphism of genes associated with folate metabolism is considered a potential factor that may influence the risk of colorectal cancer and response to treatment.

Methylenetetrahydrofolate reductase (MTHFR), an important enzyme in folate metabolism, has a major impact on the regulation of the folic acid pathway due to the conversion of 5, 10 methylenetetrahydrofolate (methylene-THF) to 5-methyl-THF. The most 
common polymorphisms of the MTHFR gene are C677T and A1298C, which are thought to reduce the enzyme activity leading to a decrease in methyl-THF and to be associated with hyperhomocysteinemia, particularly in folate deficiency. The $677 \mathrm{C} \rightarrow \mathrm{T}$ transition (exon 4) causes an amino acid substitution from alanine to valine at codon 222 within the catalytic region of the enzyme, resulting in MTHFR with decreased activity (Jacques et al., 1996). The heterozygote and homozygous variant of C677T were shown to have 30 to $65 \%$ of enzyme activity, respectively (Frosst et al., 1995). The low enzymatic activity of the MTHFR C677T genotypic variant is associated with DNA hypomethylation, which may induce genomic instability or the derepression of tumor suppressor genes. The $1298 \mathrm{~A} \rightarrow \mathrm{C}$ substitution is within the proposed regulatory region of the methylenetetrahydrofolate reductase enzyme and may influence overall enzyme function, particularly in combination with the $677 \mathrm{C} \rightarrow \mathrm{T}$ polymorphism (Singh et al., 2005; Brockton, 2006). Several studies have reported that MTHFR variants play an important role in disease processes and the susceptibility to some disorders, including vascular diseases, neural tube defects and cancer. The association between the MTHFR gene polymorphisms and genetic susceptibility to colorectal cancer has been widely evaluated in recent studies, but the conclusions are controversial. Several studies have reported that a homozygous variant genotype of the polymorphism of MTHFRC677T is associated with an increased risk of colorectal cancer (Delgado-Enciso et al., 2001; Shannon et al., 2002). However, other studies report that individuals with the MTHFR 677TT genotype have a decreased risk of colorectal cancer (Duthie, 1999), whereas yet others observe no association between the MTHFRC677T genotype and genetic susceptibility to gastric and colorectal cancer (Vollset et al., 2007; Zeybek et al., 2007). Small sample size, various ethnic groups, diet, environment, and methodologies may be responsible for the discrepancy. In the present study, we performed a case-control study to determine whether MTHFR polymorphisms and haplotypes are associated with colorectal cancer risk in an eastern Chinese population.

\section{MATERIAL AND METHODS}

\section{Subjects}

The study population consisted of 137 patients with newly diagnosed colorectal cancer and 145 population-based controls. All CRC cases were histopathologically diagnosed as primary CRC at the Affiliated People's Hospital of Jiangsu University between January 2007 and August 2010. The patients were newly diagnosed within the preceding 6 months, and had not yet been treated by any medical treatments. Patients were excluded if they were suffering from: (a) recurrence of CRC; (b) hereditary nonpolyposis CRC; (c) familial adenomatous polyposis; (d) other tumors; (e) severe digestive tract diseases over $2 \mathrm{y}$; and (f) diabetes, fatty liver, hepatic cirrhosis, metabolism syndrome, and severe cardiovascular diseases. The control group was randomly recruited by general practitioners covering the same geographical region as the colorectal cancer patients. Control participants with a history of cancer, previous radiotherapy or chemotherapy, heart disease, diabetes, and thrombosis were excluded from this study. The study was approved by the Institutional Review Board of the Affiliated People's Hospital of Jiangsu University in Zhenjiang, China. At the time of their peripheral blood collections, all case and control subjects gave their informed consent to participate in this study. 


\section{Genotyping and determination of folate and homocysteine concentrations}

The genotypes for the C677T (rs1801133) and A1298C(rs1801131) polymorphism of MTHFR were determined by polymerase chain reaction (PCR)-restriction fragment length polymorphism analysis as described by Chen et al. (1996) with minor modifications. Briefly, amplification of MTHFR C677T used 2 primers, [F]:5'-GAAGCAGGGAGCTTTGAGG-3' and $[\mathrm{R}]: 5^{\prime}$-ACGATGGGGCAAGTGATG-3'. The PCR product (152 bp) was digested with 10 U HinfI restriction enzyme (Fermentas Life Sciences) in a $20 \mu \mathrm{L}$ reaction mixture containing 5 $\mu \mathrm{L}$ PCR fragments and $2 \mu \mathrm{L} 10 \mathrm{X}$ buffer at $37^{\circ} \mathrm{C}$ overnight. Digestion products were visualized after electrophoresis on $10 \%$ polyacrylamide gels with ethidium bromide. TT homozygotes showed two fragments of 98 and $54 \mathrm{bp}$; CT heterozygotes showed three fragments of 152, 98, and $54 \mathrm{bp}$; wild-type homozygotes (CC) showed only one band of $152 \mathrm{bp}$. Amplification of MTHFR A1298C used 2 primers [F]:5'-AGAGCAAGTCCCCCAAGGA-3' and [R]:5'-CTTT GTGACCATTCCGGTTTG-3'.The PCR product of A1298C (123 bp) was digested with $10 \mathrm{U}$ MboII restriction enzyme (Fermentas Life Sciences) in a $20-\mu \mathrm{L}$ reaction mixture containing 5 $\mu \mathrm{L}$ PCR fragments and $2 \mu \mathrm{L} 10 \mathrm{X}$ buffer at $37^{\circ} \mathrm{C}$ overnight. Digestion products were visualized after electrophoresis on $10 \%$ polyacrylamide gels with ethidium bromide. Individuals with the CC genotype showed a band of $95 \mathrm{bp}$ and a residue of $28 \mathrm{bp}, \mathrm{AC}$ heterozygotes showed two bands of 95 and $67 \mathrm{bp}$ and three residues of $28 \mathrm{bp}$, and AA homozygotes showed a band of 67 bp and two 28-bp residual bands. The concentrations of folate and homocysteine in plasma were measured by chemiluminescent immunoassay (Bayer Diagnostics, Tarrytown, NY, USA).

\section{Statistical analysis}

The statistical significance of different variables was performed with $\chi^{2}$ or the Fisher exact test. Means were compared by the $t$ test or analysis of variance. The relative risk for the C677T and A1298C polymorphisms in the heterozygous and homozygous states were expressed as an odds ratio (OR) with $95 \% \mathrm{CI}$, using individuals who were homozygous wildtype as the reference group. The expected frequency of control genotypes was checked by the Hardy-Weinberg equilibrium test. All analyses were performed using the Statistical Package for the Social Sciences software version 12.0 (SPSS, Chicago, IL, USA).

\section{RESULTS}

The characteristics of the study population are presented in Table 1. In total, 137 cases and 145 controls were included in these analyses. No statistical differences were observed between cases and controls in the distribution of age and sex, suggesting that the frequency matching was adequate. A statistically significant difference in smoking status or drinking status was also not found between patients with colorectal cancer and healthy controls.

Figure 2 shows the results of PCR-RFLP. Table 2 shows the genotype distributions for MTHFR and their ORs and 95\% CIs in colorectal cancer. The distribution of the MTHFR C677T gene polymorphisms and MTHFR A1298C gene polymorphisms in the controls was calculated for Hardy-Weinberg equilibrium. The MTHFR C677T frequencies of CC, CT, and TT genotypes were $49.6,39.4$, and $10.9 \%$ in colorectal cancer, and $37.9,44.1$, and $17.9 \%$ in the controls, respectively. The frequencies of combination for 677 CT heterozygous and 677 TT 
homozygous observed were $30.7 \%$ in colorectal cancer and $40.0 \%$ in the controls. The MTHFR A1298C frequencies of AA, AC, and CC genotypes were 59.1, 39.4, and 1.5\% in colorectal cancer, and 44.8, 49.7, and 5.5\% in the controls, respectively. The frequencies of the combination of $1298 \mathrm{AC}$ heterozygous and $1298 \mathrm{CC}$ homozygous were $18.6 \%$ in colorectal cancer and $26.9 \%$ in the controls. Compared to the CC genotype, the TT genotype was significantly correlated with a reduced risk of colorectal cancer $(\mathrm{OR}=0.467 ; 95 \% \mathrm{CI}=0.225-0.966, \mathrm{P}=0.038)$. Similarly, compared to the MTHFR1298 AA genotype, the CC genotype was significantly correlated with a reduced risk of colorectal cancer $(\mathrm{OR}=0.192 ; 95 \% \mathrm{CI}=0.040-0.916, \mathrm{P}=0.023)$.

\begin{tabular}{|c|c|c|c|c|c|}
\hline & \multicolumn{2}{|c|}{ Cases $(\mathrm{N}=137)$} & \multicolumn{2}{|c|}{ Controls $(\mathrm{N}=145)$} & \multirow[b]{2}{*}{$\mathrm{P}$} \\
\hline & $\mathrm{N}$ & $\%$ & $\mathrm{~N}$ & $\%$ & \\
\hline \multicolumn{6}{|c|}{ Age (years) } \\
\hline$\leq 55$ & 60 & 43.8 & 66 & 45.5 & \multirow[t]{2}{*}{0.771} \\
\hline$>55$ & 77 & 56.2 & 79 & 54.5 & \\
\hline \multicolumn{6}{|l|}{ Gender } \\
\hline Male & 98 & 71.5 & 103 & 71.0 & \multirow[t]{2}{*}{0.926} \\
\hline Female & 39 & 28.5 & 42 & 29.0 & \\
\hline \multicolumn{6}{|c|}{ Smoking status } \\
\hline Ever & 97 & 70.8 & 100 & 69.0 & \multirow[t]{2}{*}{0.737} \\
\hline Never & 40 & 29.2 & 45 & 31.0 & \\
\hline \multicolumn{6}{|c|}{ Drinking status } \\
\hline Ever & 59 & 43.1 & 61 & 42.1 & \multirow[t]{2}{*}{0.866} \\
\hline Never & 78 & 56.9 & 84 & 57.9 & \\
\hline
\end{tabular}

\begin{tabular}{|c|c|c|c|c|c|c|}
\hline Polymorphism & & Cases $(\mathrm{N}=137)(\%)$ & Controls $(\mathrm{N}=145)(\%)$ & OR & $95 \% \mathrm{CI}$ & $\mathrm{P}$ \\
\hline \multirow[t]{5}{*}{677} & $\mathrm{CC}$ & $68(49.6)$ & $55(37.9)$ & & & \\
\hline & $\mathrm{CT}$ & $54(39.4)$ & $64(44.1)$ & 0.682 & $0.411-1.134$ & $0.139^{*}\left(0.157^{* *}\right)$ \\
\hline & TT & $15(10.9)$ & $26(17.9)$ & 0.467 & $0.225-0.966$ & $0.038^{*}\left(0.047^{* *}\right)$ \\
\hline & $\mathrm{C}$-allele & $190(69.3)$ & $174(60.0)$ & & & \\
\hline & T-allele & $84(30.7)$ & $116(40.0)$ & 0.663 & $0.468-0.939$ & $0.020 *(0.022 * *)$ \\
\hline \multirow{5}{*}{1298} & AA & $88(59.1)$ & $76(44.8)$ & & & \\
\hline & $\mathrm{AC}$ & $47(39.4)$ & $60(49.7)$ & 0.677 & $0.415-1.104$ & $0.117^{*}\left(0.136^{* *}\right)$ \\
\hline & $\mathrm{CC}$ & $2(1.5)$ & $9(5.5)$ & 0.192 & $0.040-0.916$ & $0.023 *(0.029 * *)$ \\
\hline & A-allele & $223(81.4)$ & $212(73.1)$ & & & \\
\hline & C-allele & $51(18.6)$ & 78 (26.9) & 0.622 & $0.417-0.927$ & $0.019 *(0.021 * *)$ \\
\hline
\end{tabular}

*Pearson chi-square. ${ }^{* *}$ Fisher exact test.

The interaction between the MTHFR 677 and 1298 polymorphisms on the risk of colorectal cancer was further examined. Table 3 shows the ORs of colorectal cancer associated with the MTHFR 677 genotypes by the MTHFR 1298 polymorphism. Although there was no evidence of interaction between these two polymorphisms, a joint effect between the MTHFR 677 and 1298 polymorphisms on risk of colorectal cancer was observed. Compared to the MTHFR677CC genotype and MTHFR1298 AA genotype, among individuals who carried both MTHFR $677 \mathrm{CC}$ and $1298 \mathrm{CC}$ genotypes, the OR of colorectal cancer was 0.103 (95\%CI, 0.012-0.900); among individuals who carried both MTHFR 677TT and 1298AC genotypes, the OR of colorectal cancer was $0.169(95 \% \mathrm{CI}=0.044-0.654)$. We also found that the $677 \mathrm{CC} / 1298 \mathrm{CC}$ or $677 \mathrm{CT} / 1298 \mathrm{CC}$ variants were rare and that $677 \mathrm{TT} / 1298 \mathrm{CC}$ genotypes were completely absent in our study populations, which is consistent with findings reported by other investigators (Weisberg et al., 1998). 
Table 3. MTHFR haplotype analysis and colorectal cancer susceptibility.

\begin{tabular}{|c|c|c|c|c|c|c|}
\hline MTHFR 677 & MTHFR 1298 & Cases $(\%)$ & Controls (\%) & OR & $95 \% \mathrm{CI}$ & $\mathrm{P}$ \\
\hline $\mathrm{CC}$ & $\mathrm{AA}$ & 50 & 31 & & & \\
\hline $\mathrm{CC}$ & $\mathrm{AC}$ & 17 & 18 & 0.586 & $0.263-1.303$ & $0.188^{*}(0.222 * *)$ \\
\hline $\mathrm{CC}$ & $\mathrm{CC}$ & 1 & 6 & 0.103 & $0.012-0.900$ & $0.015 *(0.039 * *)$ \\
\hline $\mathrm{CT}$ & AA & 26 & 30 & 0.537 & $0.269-1.072$ & $0.076^{*}\left(0.083^{* *}\right)$ \\
\hline $\mathrm{CT}$ & $\mathrm{AC}$ & 27 & 31 & 0.540 & $0.273-1.069$ & $0.076^{*}\left(0.086^{* *}\right)$ \\
\hline $\mathrm{CT}$ & $\mathrm{CC}$ & 1 & 3 & 0.207 & $0.021-2.076$ & $0.143 *(0.297 * *)$ \\
\hline $\mathrm{TT}$ & AA & 12 & 15 & 0.496 & $0.205-1.198$ & $0.116^{*}\left(0.124^{* *}\right)$ \\
\hline TT & $\mathrm{AC}$ & 3 & 11 & 0.169 & $0.044-0.654$ & $0.005^{*}\left(0.008^{* *}\right)$ \\
\hline $\mathrm{TT}$ & $\mathrm{CC}$ & 0 & 0 & - & - & - \\
\hline
\end{tabular}

*Pearson chi-square. ${ }^{* *}$ Fisher exact test.

Finally, we analyzed the relationships between the plasma levels of folate and MTHFR genotypes. MTHFR 677TT+CT genotypes showed a lower plasma folate concentration than those with the MTHFR 677CC genotype $(\mathrm{P}<0.05)$. MTHFR 1298AC+CC genotypes showed a lower plasma folate concentration than those with the MTHFR 1298AA genotype $(\mathrm{P}<0.05)$ (Figure 1).
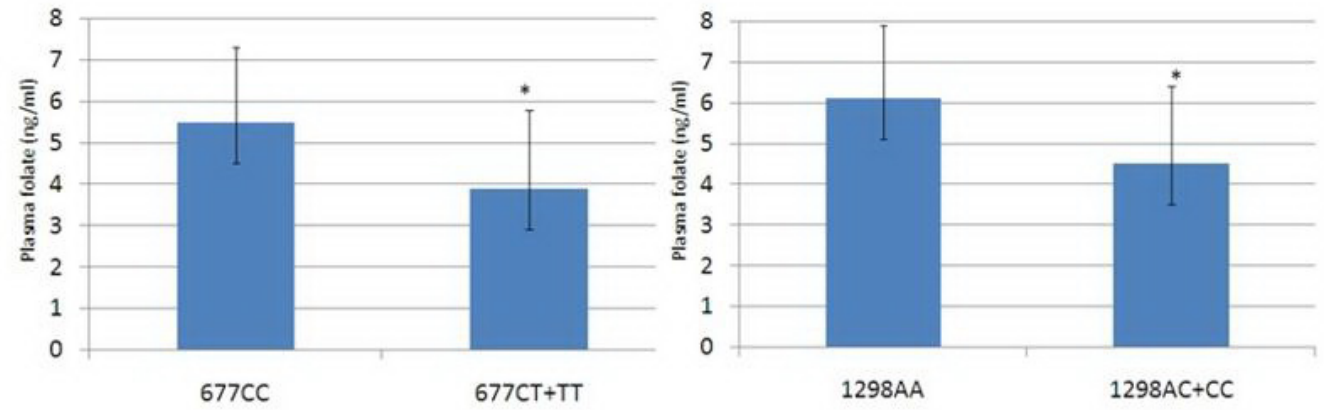

Figure 1. Correlation between plasma folate and genotype in colorectal cancer patients. Bars represent folate levels in the MTHFR polymorphisms. MTHFR $677 \mathrm{CT}+\mathrm{TT}$ genotype shows significantly lower folate concentrations than does the $\mathrm{CC}$ genotype. ${ }^{*} \mathrm{P}<0.05$. MTHFR $1298 \mathrm{AC}+\mathrm{CC}$ genotype shows significantly lower folate concentrations than does the AA genotype. ${ }^{*} \mathrm{P}<0.05$.

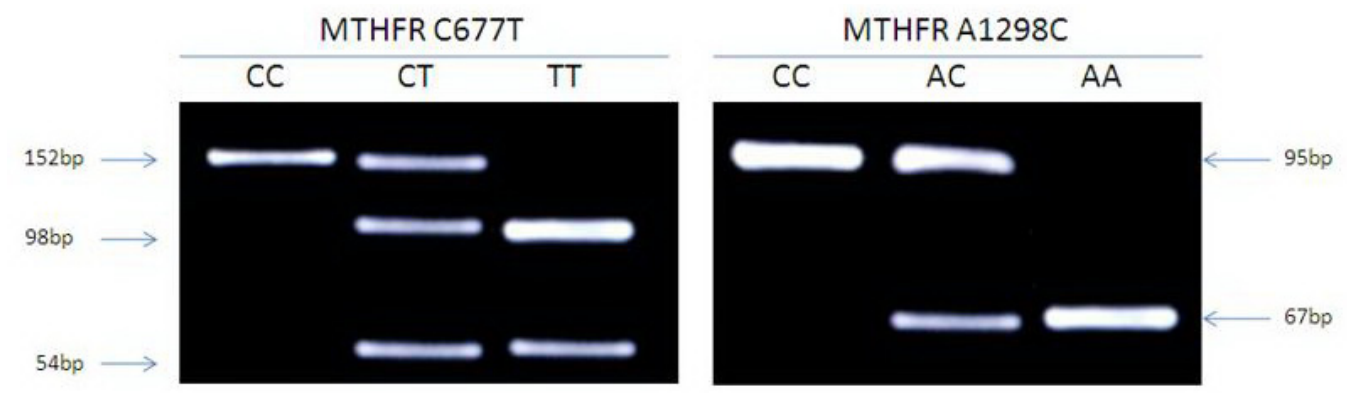

Figure 2. Results of the polymerase chain reaction-restriction fragment length polymorphism assay of the MTHFR C677T and A1298C genotypes. 


\section{DISCUSSION}

Colorectal cancer is a complex disease influenced by genetic and environmental factors and their interactions (Cheah, 2009). Growing evidence suggests that some polymorphisms in the MTHFR gene contribute to sporadic colorectal cancer. One-carbon units that folate provides are required for various biological reactions, such as DNA synthesis, repair, and methylation. MTHFR is an important enzymes in one-carbon metabolism, and its polymorphisms influence plasma folate and homocysteine levels, which have been found to be associated with the risk and the progression of colorectal cancer. Methylation of genomic DNA relies on an adequate intake of folate, and folate status may influence the risk for colorectal carcinogenesis by inducing aberrant DNA methylation, DNA instability, and alteration of gene expression (Duthie, 1999, 2000; Hayashi et al., 2007).

In the present case-control study, we employed a candidate-gene approach to investigate the associations between two polymorphisms in the MTHFR gene and the risk for colorectal cancer. The results of this study showed a significantly different distribution of some alleles ( $\mathrm{T}$ allele in MTHFR C677T, C allele in MTHFR A1298C) between cases and controls. We also observed a significant association of two polymorphisms in MTHFR with susceptibility to colorectal cancer. In particular, individuals carrying MTHFR 677TT and MTHFR 1298CC genotypes were at a lower risk for colorectal cancer, while other genotypes were not associated with colorectal cancer risk. Furthermore, the haplotype analysis based on the two investigated MTHFR polymorphisms (C677T and A1298C) showed that haplotypes $677 \mathrm{CC} / 1298 \mathrm{CC}$ and $677 \mathrm{TT} / 1298 \mathrm{AC}$ were less common in cases than controls. The haplotypes, when compared to the most common haplotype $677 \mathrm{CC} / 1298 \mathrm{AA}$, were associated with a decreased risk for colorectal cancer.

The association observed between the SNPs in the MTHFR gene and susceptibility to colorectal cancer has been inconsistent. A few studies found that the MTHFR polymorphism C677T does not affect the risk of colorectal cancer. However, the majority of studies published provided good evidence that homozygosity for the T-allele is associated with a modest but significantly reduced risk for this cancer. A meta-analysis (Huang et al., 2007) of 20 studies including 10,131 colorectal cancer patients and 15,362 controls, suggested that the 677T allele provides a protective effect against colorectal cancer risk. Moreover, Taioli et al. published a HuGE review on the MTHFR C677T polymorphism, in which the 677TT genotype appeared to be associated with a reduced risk for colorectal cancer (Taioli et al., 2009). In the present study, the association observed between the C677T SNP in the MTHFR gene and susceptibility to colorectal cancer is in agreement with the majority of studies published. The association of MTHFR A1298C polymorphism with CRC has not been extensively studied. A casecontrol study of Japanese population observed no association between A1298C polymorphism and risk of colorectal cancer (Kono and Chen, 2005). Only one published report from India is available on the association of CRC and MTHFR 1298 polymorphism. Wang et al. (2006) in a case-control study in colorectal cancer patients in an Indian population reported that the MTHFR $1298 \mathrm{C}$ allele is common among Indians and the MTHFR 1298 CC genotype was significantly associated with decreased colon and rectal cancer risk. In the present study, we found that $1298 \mathrm{CC}$ had an OR of 0.192 with a significant P value, suggesting that the 1298 $\mathrm{CC}$ genotype appeared to be associated with a reduced risk for colorectal cancer.

To the best of our knowledge, only two studies have examined MTHFR polymor- 
phisms and the risk of colorectal cancer in a Chinese Han population. However, conclusions of those studies were controversial. One study by Jiang et al. (2005), involving 128 colorectal cancer patients and 343 controls, found that the MTHFR 677TT genotype showed a significantly reduced risk of colon cancer compared to the wild type $(\mathrm{OR}=0.22,95 \% \mathrm{CI}=0.50-0.98)$, and that $1298 \mathrm{C}$ allele-carrier showed an inverse association with the risk of rectal cancer compared to the wild-type $(\mathrm{OR}=0.52,95 \% \mathrm{CI}=0.28-0.98)$. Another study (Cao et al., 2008) included 315 colorectal cancer and 371 controls, where the results from that study showed that individuals who had C677T T/T and A1298C A/A genotypes were at an increased risk of developing colon cancer (adjusted $\mathrm{OR}=2.64,95 \% \mathrm{CI}=1.20-5.81$ ) compared to those with C677T $\mathrm{C}$ allele and A1298C A/A genotypes among males. Our results showed a tendency toward lowered colorectal cancer risk in individuals with the MTHFR 677TT genotype and MTHFR 1298CC genotype. The conflicting results regarding the associations between MTHFR C677T polymorphisms and risks for colorectal cancer may be due to differences in regional dietary and local carcinogen exposures. It has been suggested that folate helps prevent cancer, the relationship between folate and colorectal cancer is complicated because folic acid has been shown to have a dual role in cancer development; specifically, low folate ingestion protects against early carcinogenesis, but high folate intake promotes carcinogenesis (Kim, 2003).

However, there were some limitations in our study. First, only 137 CRC patients and 145 controls were included in the study. Second, it would also be very interesting to see the distribution of the MTHR polymorphism in different stages. Finally, we did not determine dietary folate intake in the case groups and controls, and we also did not collect detailed data on the risk factors of colorectal cancer. Therefore, we cannot add to the debate on the relationship between gene-environment interactions.

In conclusion, this study demonstrated that subjects with the variant MTHFR 677TT genotype and MTHFR 1298CC appeared to have a significantly lower risk for colorectal cancer than those with the MTHFR 677CC genotype and MTHFR1298AA genotype. The haplotypes, $677 \mathrm{CC} / 1298 \mathrm{CC}$ and $677 \mathrm{TT} / 1298 \mathrm{AC}$, when compared to the most common haplotype $677 \mathrm{CC} / 1298 \mathrm{AA}$, were associated with a decreased risk for colorectal cancer. The plasma folate level was influenced by the MTHFR genotype. The results of this study provide a genetic basis for the hypothesis that folate metabolism may play a role in the carcinogenesis of colorectal cancer in the Chinese Han population.

\section{REFERENCES}

Boyle P and Langman JS (2000). ABC of colorectal cancer: Epidemiology. BMJ 321: 805-808.

Boyle P and Ferlay J (2005). Cancer incidence and mortality in Europe, 2004. Ann. Oncol. 16: 481-488.

Brockton NT (2006). Localized depletion: the key to colorectal cancer risk mediated by MTHFR genotype and folate? Cancer Causes Control 17: 1005-1016.

Cao HX, Gao CM, Takezaki T, Wu JZ, et al. (2008). Genetic polymorphisms of methylenetetrahydrofolate reductase and susceptibility to colorectal cancer. Asian Pac. J. Cancer Prev. 9: 203-208.

Cheah PY (2009). Recent advances in colorectal cancer genetics and diagnostics. Crit. Rev. Oncol. Hematol. 69: 45-55.

Chen J, Giovannucci E, Kelsey K, Rimm EB, et al. (1996). A methylenetetrahydrofolate reductase polymorphism and the risk of colorectal cancer. Cancer Res. 56: 4862-4864.

Choi SW and Mason JB (2000). Folate and carcinogenesis: an integrated scheme. J. Nutr. 130: 129-132.

Coleman MP, Quaresma M, Berrino F, Lutz JM, et al. (2008). Cancer survival in five continents: a worldwide populationbased study (CONCORD). Lancet Oncol. 9: 730-756.

Delgado-Enciso I, Martinez-Garza SG, Rojas-Martinez A, Ortiz-Lopez R, et al. (2001). 677T mutation of the MTHFR gene in adenomas and colorectal cancer in a population sample from the Northeastern Mexico. Preliminary results. 
Rev. Gastroenterol. Mex. 66: 32-37.

Duthie SJ (1999). Folic acid deficiency and cancer: mechanisms of DNA instability. Br. Med. Bull. 55: 578-592.

Duthie SJ, Narayanan S, Blum S, Pirie L, et al. (2000). Folate deficiency in vitro induces uracil misincorporation and DNA hypomethylation and inhibits DNA excision repair in immortalized normal human colon epithelial cells. Nutr. Cancer 37: 245-251.

Eichholzer M, Luthy J, Moser U and Fowler B (2001). Folate and the risk of colorectal, breast and cervix cancer: the epidemiological evidence. Swiss. Med. Wkly. 131: 539-549.

Frosst P, Blom HJ, Milos R, Goyette P, et al. (1995). A candidate genetic risk factor for vascular disease: a common mutation in methylenetetrahydrofolate reductase. Nat. Genet. 10: 111-113.

Hayashi I, Sohn KJ, Stempak JM, Croxford R, et al. (2007). Folate deficiency induces cell-specific changes in the steadystate transcript levels of genes involved in folate metabolism and 1-carbon transfer reactions in human colonic epithelial cells. J. Nutr. 137: 607-613.

Huang Y, Han S, Li Y, Mao Y, et al. (2007). Different roles of MTHFR C677T and A1298C polymorphisms in colorectal adenoma and colorectal cancer: a meta-analysis. J. Hum. Genet. 52: 73-85.

Jacques PF, Bostom AG, Williams RR, Ellison RC, et al. (1996). Relation between folate status, a common mutation in methylenetetrahydrofolate reductase, and plasma homocysteine concentrations. Circulation 93: 7-9.

Jemal A, Siegel R, Ward E, Murray T, et al. (2007). Cancer statistics, 2007. CA Cancer J. Clin. 57: 43-66.

Jiang Q, Chen K, Ma X, Li Q, et al. (2005). Diets, polymorphisms of methylenetetrahydrofolate reductase, and the susceptibility of colon cancer and rectal cancer. Cancer Detect. Prev. 29: 146-154.

Kim YI (2003). Role of folate in colon cancer development and progression. J. Nutr. 133: 3731S-3739S.

Kono S and Chen K (2005). Genetic polymorphisms of methylenetetrahydrofolate reductase and colorectal cancer and adenoma. Cancer Sci. 96: 535-542.

La Vecchia C, Negri E, Pelucchi C and Franceschi S (2002). Dietary folate and colorectal cancer. Int. J. Cancer 102: 545-547.

Larsson SC, Giovannucci E and Wolk A (2006a). Folate intake, MTHFR polymorphisms, and risk of esophageal, gastric, and pancreatic cancer: a meta-analysis. Gastroenterology 131: 1271-1283.

Larsson SC, Hakansson N, Giovannucci E and Wolk A (2006b). Folate intake and pancreatic cancer incidence: a prospective study of Swedish women and men. J. Natl. Cancer Inst. 98: 407-413.

Parkin DM, Bray F, Ferlay J and Pisani P (2001). Estimating the world cancer burden: Globocan 2000. Int. J. Cancer 94: 153-156.

Shannon B, Gnanasampanthan S, Beilby J and Iacopetta B (2002). A polymorphism in the methylenetetrahydrofolate reductase gene predisposes to colorectal cancers with microsatellite instability. Gut 50: 520-524.

Singh K, Singh SK, Sah R, Singh I, et al. (2005). Mutation C677T in the methylenetetrahydrofolate reductase gene is associated with male infertility in an Indian population. Int. J. Androl. 28: 115-119.

Song F, He M, Li H, Qian B, et al. (2008). A cancer incidence survey in Tianjin: the third largest city in China-between 1981 and 2000. Cancer Causes Control 19: 443-450.

Taioli E, Garza MA, Ahn YO, Bishop DT, et al. (2009). Meta- and pooled analyses of the methylenetetrahydrofolate reductase (MTHFR) C677T polymorphism and colorectal cancer: a HuGE-GSEC review. Am. J. Epidemiol. 170: 1207-1221.

Vollset SE, Igland J, Jenab M, Fredriksen A, et al. (2007). The association of gastric cancer risk with plasma folate, cobalamin, and methylenetetrahydrofolate reductase polymorphisms in the European Prospective Investigation into Cancer and Nutrition. Cancer Epidemiol. Biomarkers Prev. 16: 2416-2424.

Wang J, Gajalakshmi V, Jiang J, Kuriki K, et al. (2006). Associations between 5,10-methylenetetrahydrofolate reductase codon 677 and 1298 genetic polymorphisms and environmental factors with reference to susceptibility to colorectal cancer: a case-control study in an Indian population. Int. J. Cancer 118: 991-997.

Wei YS, Lu JC, Wang L, Lan P, et al. (2009). Risk factors for sporadic colorectal cancer in southern Chinese. World J. Gastroenterol. 15: 2526-2530.

Weisberg I, Tran P, Christensen B, Sibani S, et al. (1998). A second genetic polymorphism in methylenetetrahydrofolate reductase (MTHFR) associated with decreased enzyme activity. Mol. Genet. Metab. 64: 169-172.

Zeybek U, Yaylim I, Yilmaz H, Agachan B, et al. (2007). Methylenetetrahydrofolate reductase C677T polymorphism in patients with gastric and colorectal cancer. Cell Biochem. Funct. 25: 419-422. 